\title{
Lesional mesial temporal lobe epilepsy and limited resections: prognostic factors and outcome
}

\author{
H Clusmann, T Kral, E Fackeldey, I Blümcke, C Helmstaedter, J von Oertzen, H Urbach, J Schramm
}

J Neurol Neurosurg Psychiatry 2004;75:1589-1596. doi: 10.1136/jnnp.2003.024208

See end of article for authors' affiliations

Correspondence to:

Dr H Clusmann,

Neurochirurgische

Universitätsklinik,

Sigmund-Freud-Str 25,

53105 Bonn, Germany;

hans.clusmann@

ukb.uni-bonn.de

Received 23 August 2003

Revised 4 January 2004

Accepted 3 February 2004

\begin{abstract}
Objectives: To evaluate the influence of clinical, investigational, surgical, and histopathological factors on postoperative seizure relief in patients with mesial temporal lobe epilepsy (MTLE) due to lesions other than ammonshornsclerosis (AHS).

Methods: Of 738 patients operated for TLE, 78 patients underwent limited resections for lesional MTLE (1990-2000). Seventy four patients with a follow up of more than one year were included. The preoperative clinical, neuropsychological, electroencephalogram, and neuroimaging characteristics were prospectively collected in a database. The histopathological material was re-examined.

Results: The mean follow up was 49 months. Fifty eight patients were classified as seizure free $\mathbf{7 8 . 4 \%}$ Class I), and six as almost seizure free (8.1\% Class II), grouped together as satisfactory seizure control (64 patients, $86.5 \%$ ). Five patients (6.8\%) were categorised in Classes III and IV, respectively. These were grouped as unsatisfactory seizure control (10 patients, 13.5\%). Surgical procedures were: 32 amygdalohippocampectomies $(\mathrm{AH}), 17$ partial anterior $\mathrm{AH}, 15 \mathrm{AH}$ plus polar resection, seven $\mathrm{AH}$ plus basal resection, and three $\mathrm{AH}$ plus extended temporal lesionectomy. There was no mortality and $2.7 \%$ mild permanent morbidity. Seizure relief did neither differ significantly with these approaches, nor with different classes of pathological findings (43 developmental tumours, 12 glial tumours, 10 dysplasias, and nine others). Even operation of dysplasias resulted in $80 \%$ satisfactory seizure control. Seizure onset during childhood proved to be a negative predictor for seizure relief $(p=0.020)$. MRI revealed 73 suspected lesions $(98.6 \%)$, one dysembryoplastic neuroepithelial tumour was missed, in four cases no structural abnormalities could be confirmed with histopathological exam. Additionally, multifactorial regression revealed the factors "seizure onset after 10 years of age", "presence of complex partial seizures", "absence of a neurological deficit", and a "correlating neuropsychological deficit" as predictive for satisfactory seizure control.

Conclusions: "Preoperative tailoring" resulting in limit resections has proven to be safe and to provide a very good chance for satisfactory seizure relief in patients with lesional MTLE.
\end{abstract}

$\mathrm{S}$ uccess in the treatment of refractory temporal lobe epilepsy (TLE) has improved since the introduction of elaborate electroencephalogram (EEG) telemetry and high resolution magnetic resonance imaging (MRI) during the last two decades. ${ }^{1-6}$ Due to the widely accepted application of modern microneurosurgical techniques, epilepsy surgery has become safe and promising. ${ }^{78}$ Surgical treatment has proven to be more effective with regard to seizure control than pharmacological treatment only. ${ }^{9}{ }^{10}$ A majority of series have reported on the surgical treatment of TLE associated with mesial temporal sclerosis (MTS). ${ }^{3511}{ }^{12}$ Some studies examined TLE with different underlying pathologies. ${ }^{4613}$ Only a few studies have exclusively dealt with TLE associated with lesions other than MTS-for example, tumours or dysplasias, which is thought to represent a clinically distinct group. ${ }^{14}$ Hennessy and coworkers described a series of 80 patients with temporal foreign tissue lesions and reviewed the literature on this topic. ${ }^{15}$ There are no data available on prognostic factors and seizure outcome with different kinds of mesiotemporal lesions and refractory MTLE.

Moreover, the choice of surgical approach and resection planning is a matter of discussion. Anterior temporal lobectomy is still the most frequently applied standard resection for treating TLE. ${ }^{141617}$ Nevertheless, beneficial effects have been shown with respect to postoperative neuropsychological performances in patients after limited resections for TLE-for example, lesionectomy and amygdalohippocampectomy (AH). ${ }^{6}{ }^{18}$ During the last 10 years we have practiced a concept of "preoperative tailoring". This implies that a resection is limited to the MRI documented lesion plus the adjacent presumed epileptogenic tissue, partly detected with invasive EEG monitoring. ${ }^{19}$

We therefore analysed predictive factors and outcome in our consecutive patient series operated for lesional MTLE with limited resections between 1990 and 2000.

\section{PATIENTS AND METHODS \\ Patients}

From 1990 to 2000, a total of 738 different temporal resections were performed for medically intractable TLE in the Epilepsy Surgery Program, University of Bonn. In 335 patients resection was done for MTS. Seventy eight patients were operated for other mesial temporal lesions during the respective period. Minimal requirements for inclusion into the study were: complete clinical and electrophysiological data sets, a mesiotemporal lesion other than MTS either suspected by MRI or diagnosed with histopathological exam, "preoperatively tailored" non-lobar resection, and follow up of at least 12 months. Features of ammonshornsclerosis obtained with histopathology were only

Abbreviations: $\mathrm{AH}$, amygdalohippocampectomy; $\mathrm{AHS}$, ammonshornsclerosis; DNT, dysembryoplastic neuroepithelial tumour; $E E G$, electroencephalogram; MRI, magnetic resonance imaging; MTLE, mesial temporal lobe epilepsy; MTS, mesial temporal sclerosis; TLE, temporal lobe epilepsy. 
accepted as secondary findings in cases with predominant other mesiotemporal lesion on MRI. Four patients had to be excluded due to lack of any follow up information, resulting in a total of 74 patients. Of these, three patients had undergone previous operations or biopsies, but without presurgical evaluation of their epilepsies. A vagus nerve stimulator was implanted later in one patient with persisting seizures. Forty five patients in this study have also been incorporated in previous investigations. ${ }^{6} 132021$

\section{Presurgical evaluation}

All patients had well documented chronic and medically intractable TLE for more than one year and had adequate trials of at least two first line antiepileptic drugs, before they were referred to presurgical evaluation. All patients underwent continuous non-invasive video scalp EEG monitoring, including sphenoidal electrodes to determine ictal and interictal focal activity. Data used for this analysis comprised the following clinical and demographic information: age at epilepsy manifestation, duration of epilepsy, age at operation, febrile seizures, other medical history, neurological status, seizure types, and seizure frequency. For the purpose of this study, EEG data were classified as concordant to the morphological lesion if present, bilateral, discordant, or unclear/none, depending on the location of interictal specific activity (spikes, sharp waves, sharp slow waves) and the location of ictal onset (focal onset of synchronised and rhythmic EEG activity). Where there was missing information, patient charts were reviewed and data updated. Intracranial recordings were performed in 18 patients (24\%): amygdalohippocampal depth electrodes $(n=4)$, and subdural strip electrodes combined with amygdalohippocampal depth electrodes $(n=14)$. The details of invasive recordings from this centre have been previously described. ${ }^{22}{ }^{23}$ A global Wada test evaluation was only added in a few special cases-for example, to determine memory and speech features. Limited resections for mesiotemporal lesions do not normally interfere with eloquent areas and can be identically performed on both sides.

A neuropsychological testing of intelligence, attention, visual and verbal memory, language, and higher verbal and visual reasoning was routinely performed, as described before in detail..$^{18}{ }^{24}$ For the comprehensive purpose of this study, only preoperative results concerning the major factorsvisual and verbal memory-were taken into account, in order to evaluate lateralising features. All neuropsychological results were classified into six categories from 0 (far below average) to 5 (far better than average), with the difference between two categories being one standard deviation. Categories 2 and 3 were considered as range of normal average. A difference of at least one class in preoperative verbal and visual memory performance, but not between categories 2 and 3, was considered as "lateralising" to the side of the poorer performance. ${ }^{622}$

All patients had preoperative MRI, most of them at our institution, using a 1.5 Tesla unit (Gyroscan S15 or ACS-NT, Philips Medical Systems, Eindhoven, the Netherlands): $5 \mathrm{~mm}$ axial FLAIR and T2 weighted fast spin echo, $3 \mathrm{~mm}$ coronal FLAIR fast spin echo, $2 \mathrm{~mm}$ coronal T2 weighted fast spin echo, $5 \mathrm{~mm}$ coronal $\mathrm{Tl}$ weighted inversion recovery, and T1 weighted 3D gradient echo sequences with $1 \mathrm{~mm}^{3}$ large voxels. Tl weighted spin echo sequences with gadolinium diethylenetriamine pentaacetic acid (DTPA) were mostly available. MRI details from this centre have been recently published in more detail. ${ }^{25}$

\section{Surgical procedures}

The surgical approach was planned with respect to the lesion shown on MRI. Complete microsurgical resection of the morphological identifiable lesion was intended in all but four cases, where parts of the lesion extended into the basal ganglia. These parts were preserved during resection. Due to good anatomical guidance in the mesial temporal area, a reliable correlation of the intraoperative findings and the MRI data was generally possible. The resection was extended according to preoperative clinical and electrophysiological features-for example, for the decision on whether an additional complete or partial amygdalohippocampectomy should be done. All the procedures could be attributed to one of the following five categories: "complete" amygdalohippocampectomy (AH), partial (anterior) amygdalohippocampectomy (PARTAH), resection of the temporal pole plus amygdalohippocampectomy (POLAH), basal temporal resection plus amygdalohippocampectomy (BASAH), removal of a mesial lesion, which extends into the lateral temporal lobe (TEMPAH). For illustration see figure 1. The term "complete" $\mathrm{AH}$ was chosen with the understanding that the smaller part of the amygdalum containing the mesial nuclei is left behind. Furthermore, the hippocampal resection does not include the dorsal extension behind the brainstem level. AH was done as described by Yasargil $e \mathrm{al}^{26}$ : after microsurgical dissection and $3 \mathrm{~cm}$ anterior opening of the sylvian fissure with preservation of arterial and venous vessels, the temporal horn of the lateral ventricle was opened by incision of the temporal stem to an extent of approximately $15 \mathrm{~mm}$. After identification of the mesial lesion, first the caput hippocampi and lateral amygdala were resected, and additionally the uncus was removed. In a second step, we performed an en bloc resection of the corpus hippocampi together with the parahippocampal gyrus, preserving the arachnoid layers covering the cisterna ambiens. Lastly, the vessels in the hippocampal fissure were dissected, coagulated, and cut. Lateral resection border is the collateral sulcus, the posterior resection should reach the middle or even dorsal brainstem level. If the mesial temporal anatomy was difficult to identify because of tumour infiltration, the dissection and resection were at least partly, if not completely, done with ultrasonic aspiration. For 17
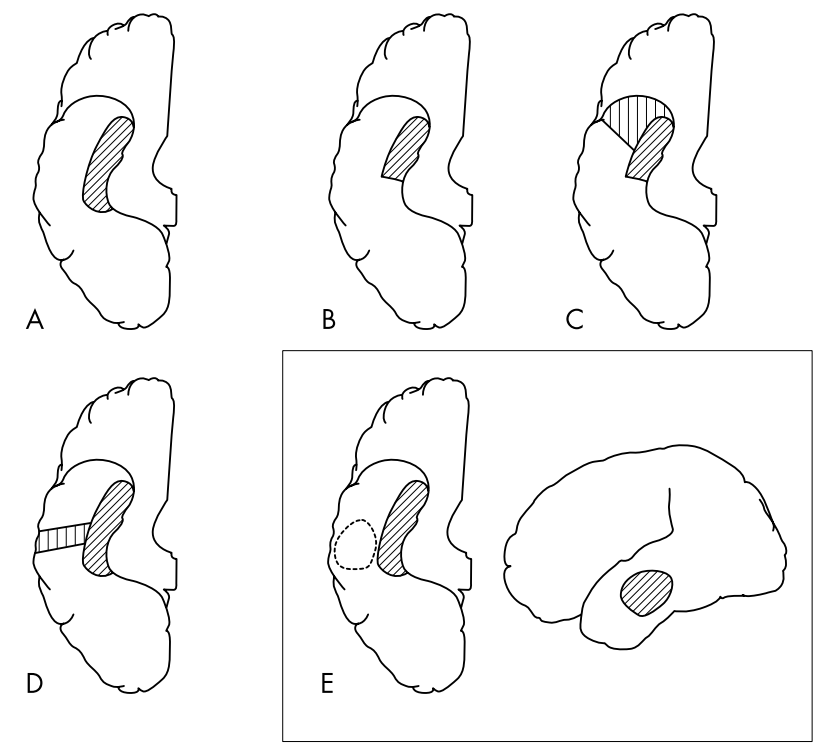

Figure 1 Schematic drawings of the five different classes of limited surgical approaches for the treatment of lesional mesial temporal lobe epilepsy (MTLE). (A) "Complete" amygdalohippocampectomy (AH); (B) Partial (anterior) amygdalohippocampectomy (PARTAH); (C) Amygdalohippocampectomy and resection of the temporal pole (POLAH); (D) Amygdalohippocampectomy and temporal basal resection (BASAH); (E) Amygdalohippocampectomy and extended lateral lesionectomy (TEMPAH). 
PARTAH, we used the transsylvian approach to an extent of approximately $2.5 \mathrm{~cm}$, took the transventricular route, and resected the lesion in the area of uncus parahippocampalis, amygdala, and caput hippocampi. The corpus hippocampi was preserved in these patients. For AH and PARTAH we preferred frontotemporal (pterional) craniotomies. In 15 patients an anterior temporal craniotomy and subsequent resection of the temporal pole was done. Then resection of the mesial lesion was completed through this approach, without opening the sylvian fissure (POLAH). With this approach it is, of course, not possible to perform extensive dorsal hippocampectomies, which was never intended when this approach was chosen. Seven lesions extending from mesial temporal areas towards basal temporal cortex were removed after a dorsal and basal temporal craniotomy. With resection of the lateral and basal parts of the lesion, we followed this extended subtemporal route to remove the hippocampal and parahippocampal area (BASAH). If there was a larger extension of a mesial lesion towards lateral parts of the temporal lobe $(n=3)$, we removed the lateral parts first and used this "transcortical" approach to identify the temporal horn of the ventricle, in order to complete the mesial resection (TEMPAH).

\section{Histological exam}

The resected specimens were histopathologically examined using standard neuropathological techniques that have been previously described. ${ }^{27}$ Haematoxylin and eosin stained sections were available for all cases. Mostly, Nissl stains and combined hematoxylin eosin luxol fast blue stains were also available. In selected cases, additional stains were performed-for example, immunohistochemical stains for the stem cell marker CD34 in developmental tumours. ${ }^{28}$ Tumours were classified according to the revised World Health Organization (WHO) classification scheme of tumours of the nervous system (1993). The diagnosis of ammonshornsclerosis and dysplastic malformations was used as previously described for temporal lobe specimens in epilepsy. ${ }^{27}$ There is an ongoing discussion on the classification of dysplastic malformations, so that all different subtypes were summarised as cortical dysplasias for the purpose of this study.

\section{Follow up}

Follow up information regarding seizure outcome and neurological status was either obtained from the last regular yearly outpatient visit or from standardised telephone interviews performed by one of the authors (EF). Patients were assigned to four different seizure outcome classes based on their last postoperative seizure status (Engel 1993) ${ }^{29}$ : (I) seizure free or auras only since surgery; (II) rare seizures (no more than two per year or only non-disabling nocturnal seizures); (III) reduction of seizure frequency $>75 \%$; and (IV) $<75 \%$ reduction of seizure frequency. For further analysis, usually Class I and II outcome was summarised as satisfactory seizure control, Classes III and IV as unsatisfactory seizure control. So far, only one patient (aged 60 years at first operation in 1999) suffered from recurrence of an astrocytoma in WHO III and thus underwent reoperation.

\section{Data collection and statistical analysis}

Data were prospectively collected in a data bank and retrospectively analysed. The potentially prognostic factors were analysed with respect to the prediction of satisfactory seizure control. For unifactorial analysis between groups, mainly defined by the seizure outcome, dichotomous discrete variables were analysed using Fisher's exact test. A critical p value of 0.05 was accepted to indicate significance for two sided testing; a p value of 0.1 was accepted to indicate a trend. For multifactorial analysis, a stepwise logistic regression model was applied. Forward stepwise regression was done with critical p levels of 0.05 for inclusion and 0.1 for exclusion of factors from the model using adjusted $\chi^{2}$

Table 1 Presurgical factors and outcome in 74 MTLE patients (unifactorial analysis)*

\begin{tabular}{|c|c|c|c|c|c|c|}
\hline \multirow{3}{*}{$\begin{array}{l}\text { Factor } \\
\text { All patients }\end{array}$} & \multicolumn{6}{|c|}{ Number of patients (\%) } \\
\hline & \multirow{2}{*}{$\begin{array}{l}\mathbf{n} \\
74\end{array}$} & \multicolumn{2}{|c|}{ Class I \& II } & \multicolumn{2}{|c|}{ Class III \& IV } & \multirow[t]{2}{*}{ p Value } \\
\hline & & 64 & $(86.5)$ & 10 & (13.5) & \\
\hline Male & 42 & 38 & (90.5) & 4 & (9.5) & \\
\hline Female & 32 & 26 & (81.3) & 6 & (18.8) & 0.313 \\
\hline \multicolumn{7}{|l|}{ Clinical findings/history } \\
\hline With auras & 34 & 31 & (91.2) & 3 & (8.8) & \\
\hline Without auras & 40 & 33 & (82.5) & 7 & (17.5) & 0.326 \\
\hline With febrile seizures & 5 & 3 & $(60.0)$ & 2 & $(40.0)$ & \\
\hline Without febrile seizures & 69 & 61 & (88.4) & 8 & (11.6) & 0.132 \\
\hline With complex partial seizures & 69 & 61 & $(88.4)$ & 8 & (11.6) & \\
\hline With simple partial seizures & 5 & 3 & $(60.0)$ & 2 & $(40.0)$ & 0.132 \\
\hline With generalised seizures & 44 & 37 & (84.1) & 7 & (15.9) & \\
\hline Without generalised seizures & 30 & 27 & (90.0) & 3 & (10.0) & 0.731 \\
\hline \multicolumn{7}{|l|}{ Age at seizure onset } \\
\hline $0-10$ years age & 20 & 14 & $(70.0)$ & 6 & $(30.0)$ & \\
\hline$>10$ years age & 54 & 50 & (92.6) & 4 & (7.4) & 0.020 \\
\hline \multicolumn{7}{|l|}{ Frequency of partial seizures } \\
\hline$<5$ per month & 18 & 17 & (94.4) & 1 & $(5.6)$ & \\
\hline 6-30 per month & 38 & 34 & (89.5) & 4 & $(10.5)$ & \\
\hline$>30$ per month & 18 & 13 & $(72.2)$ & 5 & $(27.8)$ & 0.087 \\
\hline \multicolumn{7}{|l|}{ EEG telemetry } \\
\hline Invasive recordings & 18 & 14 & $(77.8)$ & 4 & $(22.2)$ & \\
\hline Non-invasive recordings & 56 & 50 & (89.3) & 6 & (10.7) & 0.244 \\
\hline \multicolumn{7}{|l|}{ Neuropsychological deficits } \\
\hline Correlate with lesion & 43 & 40 & (93.0) & 3 & (7.0) & \\
\hline $\begin{array}{l}\text { Do not correlate/ } \\
\text { bitemporal/unclear }\end{array}$ & 31 & 24 & (77.4) & 7 & $(22.6)$ & 0.083 \\
\hline $\begin{array}{l}\text { * See outcome data for explanatio } \\
\text { whereas Classes III and IV togethe } \\
\text { exact test. Comparison of } p \text { value } \\
\text { statistical significance; a p value }\end{array}$ & d & .0 & $\begin{array}{l}\text { I and } \\
\text { comes } \\
\text { factor }\end{array}$ & me & $\begin{array}{l}\text { ent satis } \\
\mathrm{s} \text { were } \\
\text { alue of }\end{array}$ & $\begin{array}{l}\text { outcomes, } \\
\text { ith Fisher's } \\
0 \text { indicates }\end{array}$ \\
\hline
\end{tabular}


Table 2 Surgical procedures, histopathological findings, and outcome in 74 patients with lesional MTLE*

\begin{tabular}{|c|c|c|c|c|c|c|}
\hline \multirow{3}{*}{$\begin{array}{l}\text { Factor } \\
\text { All procedures }\end{array}$} & \multirow{3}{*}{$\begin{array}{l}\text { Total number } \\
\text { of patients }\end{array}$} & \multicolumn{5}{|c|}{ Number of patients with outcome (\%) } \\
\hline & & \multicolumn{2}{|c|}{ Class I \& II } & \multicolumn{2}{|c|}{ Class III \& IV } & \multirow[t]{2}{*}{ p Value } \\
\hline & & 64 & (86.5) & 10 & (13.5) & \\
\hline Right operation & 32 & 30 & (93.8) & 2 & (6.3) & \\
\hline Left operation & 42 & 34 & (81.0) & 8 & (19.0) & 0.172 \\
\hline $\mathrm{AH}$ & 32 & 28 & (87.5) & 4 & (12.5) & \\
\hline Right & 15 & 15 & (100) & 0 & (0) & \\
\hline Left & 17 & 13 & (76.5) & 4 & (23.5) & 0.104 \\
\hline Ganglioglioma/DNT & 19 & 18 & (94.7) & 1 & (5.3) & \\
\hline Other fumour & 4 & 4 & $(100)$ & 0 & (0) & \\
\hline Dysplasia & 3 & 2 & (66.7) & 1 & (33.3) & \\
\hline Other/normal & 6 & 4 & (66.7) & 2 & (33.3) & \\
\hline PARTAH & 17 & 15 & (88.2) & 2 & (11.8) & \\
\hline Right & 6 & 6 & (100) & 0 & (0) & \\
\hline Left & 11 & 9 & (81.8) & 2 & (18.2) & 0.515 \\
\hline Ganglioglioma/DNT & 10 & 9 & $(90.0)$ & 1 & $(10.0)$ & \\
\hline Other tumour & 3 & 3 & $(100)$ & 0 & (0) & \\
\hline Dysplasia & 2 & 1 & (50.0) & 1 & $(50.0)$ & \\
\hline Other & 2 & 2 & $(100)$ & 0 & (0) & \\
\hline POLAH & 15 & 14 & (93.3) & 1 & (6.7) & \\
\hline Right & 6 & 6 & $(100)$ & 0 & (0) & \\
\hline Left & 9 & 8 & (88.9) & 1 & (11.1) & 1.000 \\
\hline Ganglioglioma/DNT & 8 & 8 & $(100)$ & 0 & (0) & \\
\hline Other fumour & 3 & 2 & (66.7) & 1 & (33.3) & \\
\hline Dysplasia/other & 4 & 4 & $(100)$ & 0 & (0) & \\
\hline BASAH & 7 & 6 & (85.7) & 1 & (14.3) & \\
\hline Right & 3 & 2 & (66.7) & 1 & (33.3) & \\
\hline Left & 4 & 4 & (100) & 0 & (0) & 0.429 \\
\hline Ganglioglioma/DNT & 5 & 4 & (80.0) & 1 & $(20.0)$ & \\
\hline Tumour/dysplasia & 2 & 2 & $(100)$ & 0 & (0) & \\
\hline TEMPAH & 3 & 1 & (33.3) & 2 & (66.7) & \\
\hline Dysplasia & 1 & 1 & (100) & 0 & (0) & \\
\hline Ganglioglioma/tumour & 2 & 0 & (0) & 2 & $(100)$ & \\
\hline
\end{tabular}

statistics. The result was confirmed by backward stepwise regression.

\section{RESULTS}

The mean follow up was 49 months (range 12-118 months), the median 47 months; 62 patients $(83.8 \%)$ had a follow up of more than two years. A total of $58(78.4 \%)$ patients was classified as seizure free (Class I), and six patients (8.1\%) had rare and non-disabling seizures (Class II); these classes were grouped together as satisfactory seizure control (64 patients, $86.5 \%)$. Five patients $(6.8 \%)$ were categorised in Class III, and no worthwhile improvement was reached in another five patients (6.8\% Class IV); these classes were grouped together as unsatisfactory seizure control (10 patients, $13.5 \%$ ).

Table 3 Histopathological findings and outcome in lesional MTLE*

\begin{tabular}{|c|c|c|c|c|c|c|}
\hline \multirow{3}{*}{$\begin{array}{l}\text { Diagnosis } \\
\text { Ganglioglioma/DNT }\end{array}$} & \multirow{3}{*}{$\begin{array}{l}\text { Total number } \\
\text { of patients }\end{array}$} & \multicolumn{5}{|c|}{ Number of patients with outcome (\%) } \\
\hline & & \multicolumn{2}{|c|}{ Class I \& || } & \multicolumn{2}{|c|}{ Class III \& IV } & \multirow{2}{*}{$\frac{p \text { Value }}{0.304}$} \\
\hline & & 39 & (90.7) & 4 & (9.3) & \\
\hline Ganglioglioma & 27 & 25 & (92.6) & 2 & $(7.4)$ & \\
\hline DNT & 16 & 14 & (87.5) & 2 & (12.5) & \\
\hline Other fumour & 12 & 10 & (83.3) & 2 & (16.7) & 0.661 \\
\hline Astrocytoma & 9 & 7 & (77.8) & 2 & (22.2) & \\
\hline Oligodendroglioma & 2 & 2 & $(100)$ & 0 & (0) & \\
\hline Pleom. Xanthoastro. & $\overline{1}$ & 1 & (100) & 0 & (0) & \\
\hline Dysplasia & 10 & 8 & (80.0) & 2 & $(20.0)$ & 0.617 \\
\hline Other diagnoses & 9 & 7 & (77.8) & 2 & $(22.2)$ & 0.600 \\
\hline Normal & 4 & 2 & $(50.0)$ & 2 & $(50.0)$ & \\
\hline Vascular malform. & 4 & 4 & $(100)$ & 0 & (0) & \\
\hline Gliosis & 1 & 1 & (100) & 0 & (0) & \\
\hline Dual pathology & 5 & 4 & (80.0) & 1 & $(20.0)$ & 0.527 \\
\hline Any tumour + $\mathrm{AHS}$ & 4 & 3 & $(75.0)$ & 1 & $(25.0)$ & \\
\hline DNT + dysplasia & 1 & 1 & $(100)$ & 0 & (0) & \\
\hline \multicolumn{7}{|c|}{$\begin{array}{l}\text { See Outcome Data for explanation of Engel Classification. Classes I and II combined represent satisfactory } \\
\text { outcomes, whereas Classes III and IV together represent unsatisfactory outcomes. Statistical analyses were done } \\
\text { with Fisher's exact test. Comparison of p values: histological diagnosis compared with the other diagnoses-for } \\
\text { example, dual pathology compared to "no dual pathology". } \\
\text { AHS, ammonshornsclerosis; DNT, dysembryoplastic neuroepithelial tumour. }\end{array}$} \\
\hline
\end{tabular}




\section{Clinical variables and seizure outcome}

Demographic and relevant clinical data and its association with seizure outcome are summarised in table 1. Male patients attained insignificantly better results. The presence of the clinical items "neurological impairment or retardation", "history of febrile seizures", and "seizure types" did not reveal significant differences with respect to seizure outcome upon univariate testing. A history of febrile seizures was rare in this study group (five patients, 6.7\%). The mean age at seizure onset was 17 years (range $0.5-58$ years), the median 15.5 years. The mean duration of the epilepsy was 15 years (range $1-44$ years), the median 12 years. Results were significantly worse in the subgroup of patients with epilepsy manifestation during childhood ( $<10$ years of age) compared with the patients with later onset. The duration of the epileptic disorder did not correlate with the success of epilepsy surgery. There was only a trend of better seizure control with lower frequencies of partial seizures, whereas the frequency of generalised seizures was virtually equal with satisfactory and unsatisfactory outcomes.

Interictal and ictal EEG recordings with video EEG monitoring revealed specific EEG activity confined to the temporal area ipsilaterally to the suspected lesion in 67 patients (90.5\%). Epileptiform EEG activity was recorded in three patients in ipsilateral extratemporal areas, two showed bilateral spikes, and two patients had a normal EEG. All of these seven patients with "atypical" EEG patterns experienced satisfactory seizure control (five patients in Engel Class I; two patients in Engel Class II) (table 1).

Normal neuropsychological results were obtained from 10 patients. Atypical hemispherical dominance was found in one patient. Twenty eight patients showed lateralising results for an affection of the left temporal lobe, 22 patients had a neuropsychological profile lateralising to the right. Bitemporal impairments were documented in nine patients. Results were uncertain in five patients. Comparing seizure outcome and neuropsychological findings, we found a trend upon univariate analysis, that the presence of neuropsychological impairments lateralising to the side of surgery was associated with better seizure control (table 1).

Magnetic resonance investigations detected structural lesions in 73 patients (98.6\%); in one patient a dysembryoplastic neuroepithelial tumour (DNT) was not preoperatively seen on MRI. Of 53 cases preoperatively classified as unspecific "lesions", 22 turned out to be gangliogliomas, 10 DNTs, 10 dysplasias, six astrocytomas, one gliosis, one cavernoma, and in three cases no histopathological abnormalities were detected. Four of five incorrect MR diagnoses occurred before 1994 .

\section{Resection strategies}

The results of the analysis of surgical approaches, laterality, and diagnoses with respect to seizure outcome are shown in table 2. Generally, comparable rates of seizure relief were obtained with the different surgical approaches. Only with TEMPAH were results not as satisfactory, but the number of only three patients is too small to draw any conclusions. All right sided $\mathrm{AH}, \mathrm{PARTAH}$, and POLAH lead to complete seizure relief, although this was not significant for any of the approaches. With AH, PARTAH, and POLAH results were extraordinarily satisfying for treatment of gangliogliomas or DNTs (90-100\% Class I, II outcomes). Similar good results were obtained with $\mathrm{AH}$ and PARTAH for other tumours (table 2).

Permanent neurological deficits occurred in two patients $(2.7 \%)$ : one patient showed a persisting mild hemiparesis and hemianopia due to venous infarction after introperative bleeding from the $\mathrm{V}$ basalis Rosenthal, the other patient showed a hemianopia due to manipulation of a lesion adjacent to the optical tract. Temporary neurological deficits were seen in seven patients $(9.5 \%)$ : five patients showed a temporary dysphasia, in one patient accompanied by a mild hemiparesis, which had resolved at discharge from hospital. Two patients experienced typical infratentorial haemorrhages remote from the site of surgery, but symptoms were mild and resolved before the patients were discharged. Surgical postoperative complications were seen in five patients $(6.8 \%)$ : one developed a cyst in the resection cavity and required implantation of a shunt system, one patient developed a hygroma and later a bone infection, so that the bone flap was removed, one patient had a deep vein thrombosis and mild pulmonary embolism, and two patients developed meningitis without positive bacterial culture.

\section{Histopathology}

Histopathological diagnoses and respective seizure relief rates are presented in table 3. All 16 DNTs were in WHO Grade I, of 27 gangliogliomas two were in WHO Grade II, 25 were in WHO Grade I. We diagnosed 12 glial tumours, of which five were in WHO Grade I, four in WHO Grade II, and three in WHO Grade III. Success rates were not significantly different with different histopathological diagnoses. It is interesting to note, that even dysplastic malformations were operated with $80 \%$ satisfactory seizure control. The only disappointing results $(50 \%$ satisfactory seizure relief) were found when the specimens did not reveal pathological findings upon histopathological evaluation. Dual pathology was found in five patients $(6.8 \%)$, and outcome was not significantly different to the rest of the study group (table 3 ).

\section{Multifactorial prediction of seizure relief}

Lesions demonstrated by MRI are known to be strong positive predictors for satisfactory seizure relief. Thus, because of the mode of patient selection, only additional predictive or supportive factors could be screened for in this study group. Entering clinical and surgical factors in a regression analysis, a model with four additional factors independently supportive for satisfactory seizure control was found (table 4): seizure onset after 10 years of age, presence of complex partial seizures, absence of a neurological deficit or developmental retardation, and a neuropsychological deficit lateralising to the side of surgery. The same analysis with complete seizure relief (Engel Class I) as dependent variable only confirmed the factors "seizure onset after 10 years of age" and "absence of a neurological deficit".

Table 4 Multifactorial model of predictive factors for satisfactory or unsatisfactory seizure control: results of a stepwise regression analysis*

\begin{tabular}{lccl}
\hline Factors & Odds ratio & $\mathbf{9 5 \%} \mathrm{Cl}+$ & $\mathbf{p ~ V a l u e}$ \\
\hline Age at seizure onset & 0.323 & $0.084-0.414$ & 0.004 \\
Complex partial & 0.257 & $0.061-0.640$ & 0.018 \\
seizures & & & \\
Neurological deficit & -0.254 & $-0.334-0.027$ & 0.022 \\
Correlating & 0.226 & $0.010-0.304$ & 0.037 \\
neuropsychology & & & \\
\hline
\end{tabular}

*Stepwise forward regression analysis with backward confirmation, critical $p$ value for inclusion 0.05 , and 0.1 for exclusion: predictors for satisfactory postoperative seizure control (Engel Class I, II): seizure onset after 10 years of age, presence of complex partial seizures, absence of a neurological deficit, and a correlating neuropsychological profile. The following factors were not included in the model: febrile seizures, age at operation, duration of epilepsy, side, sex, medical history, presence of simple partial or generalised seizures, seizure frequencies, EEG focus, histological diagnoses.

†Confidence interval from regression analysis; lower and upper limits. 


\section{DISCUSSION}

\section{Study group and demographics}

Patients with TLE due to MTS can be successfully treated by removal of either the lateral and mesial anterior temporal lobe or resection of mesial structures only. ${ }^{4{ }^{13}}$ Of 738 resections performed for TLE in this epilepsy programme between 1990 and 2000, 45.4\% were done in cases with MTS. This high percentage is concordant with previous reports on surgical treatment of TLE. ${ }^{4{ }^{11} 17}$ In contrast, Hennessy et al described foreign tissue lesions in only 80 of 234 temporal lobe specimens (34.2\%). ${ }^{15}$ A report from the Mayo Clinic described focal lesions in 38\%, whereas MTS was seen in $62 \%{ }^{4}$ Varying percentages are probably due to patient selection. ${ }^{19}$ MTLE patients with predominant mesiotemporal lesions other than AHS are a much lesser recognised subgroup. Hennessy et al described only five of 80 specimens (6.3\%) with hippocampal lesions; nine cases remained unclear. ${ }^{15}$ There are only few reports dealing with lesional MTLE, which is thought to be a clinically different entity from patients with MTS. ${ }^{16}$ There are no reports available on seizure outcome and resection strategies for the treatment of this lesional MTLE subgroup. Thus, we retrospectively analysed clinical and surgical factors in 74 patients operated for lesional MTLE between 1990 and 2000.

\section{Seizure outcome}

The high percentage of patients attaining satisfactory seizure control $(86.5 \%)$ is gratifying for a series of patients with MTLE. There have been no publications on outcome in mesial temporal lesions and MTLE so far. On the other hand, any comparison of success rates is generally difficult, mainly because of different outcome scales, different time periods, follow up duration, and statistical methods. Regarding the report of Hennessy et al with results from surgery for temporal lesions, $71 \%$ of patients achieved a one year seizure remission by five years of follow up. ${ }^{15}$ However, these data are not comparable, because this series was mainly operated before introduction of MRI, and the study group was exclusively defined by histopathological criteria. Morris et al reported seizure control in 22 of 26 patients (84.6\%) with temporal gangliogliomas. ${ }^{30}$ Raymond et al described complete seizure relief in 12 of 16 patients $(75 \%)$ operated for temporal DNTs. ${ }^{31}$ Seizure relief rates differ between $67 \%$ and $84 \%$ for the surgical treatment of MTLE with AHS in recent studies $^{4-632}$ with a median of approximately $70 \% .{ }^{17}$ Excellent results were also obtained with surgical treatment of neocortical TLE. ${ }^{33}$ Taken together, the results obtained in this subgroup of patients with mesiotemporal lesions and MTLE are promising, when compared with results from patient series with other forms of TLE.

\section{Presurgical factors}

Only five patients $(6.8 \%)$ had a history of febrile seizures, which is clearly less than approximately $40 \%$ in patients with AHS, supporting the theory of different subtypes of MTLE. ${ }^{32}$ Furthermore, we only found a low rate of learning disability, mental retardation, or neurological deficits. None of the 10 patients with mesiotemporal dysplasias showed signs of mental retardation. However, we confirmed the finding that need for special schooling and features of mental retardation are negative predictive factors for seizure control. ${ }^{15}$ Interestingly, we found significantly better seizure control in patients with seizure onset after 10 years of age. The mean age at seizure onset of 17 years corresponds well with other studies reporting on epilepsy associated temporal lesions. ${ }^{6}{ }^{15}$ Onset of habitual seizures is known to be somewhat earlier in MTLE patients with AHS (average 11-12 years). ${ }^{46}{ }^{32}$ Age at seizure onset did not correlate with different histopathological findings, as might have been expected (for example, for dysplasias). Other studies reported on beneficial results especially with early treatment of TLE in young patients. ${ }^{5} 15$ Nevertheless, early onset of symptoms may be caused by either more epileptogenicity of the lesion itself, early involvement of adjacent cortical areas, or the phenomenon of secondary epileptogenesis in patients with unknown causes of seizure susceptibility. Thus, early onset may be associated with other factors, which we were not able to document either with imaging or with respect to medical history and clinical findings.

Secondary seizure generalisation has been described as a negative predictor for seizure control, especially in patients with AHS, but was not found in this series. ${ }^{11}{ }^{17}$ However, we found a trend-that a lower preoperative frequency of partial seizures may be associated with better seizure control, confirming previous findings. ${ }^{634}$ The presence of complex partial seizures was one of the supportive predictive parameters for satisfactory seizure relief.

Because of a very low rate of patients with discordant EEG patterns, information derived from EEG recordings was not suitable to discriminate patients with a lower expectation of seizure control in this study. Other studies found a significant contribution of EEG data, but mostly in patients with mesial temporal sclerosis ${ }^{3411}$ or with normal MRI results. ${ }^{35}$ Discrepancies in non-invasive EEG monitoring may lead to the need for invasive monitoring with implanted electrodes. ${ }^{19}$

Only a few reports have described neuropsychological findings contributing to outcome prediction in epilepsy surgery: in a series of 285 patients with TLE we documented a concordant memory deficit-that is, a neuropsychological deficit lateralising to the side of surgery, to be a predictor for good seizure control. ${ }^{6}$ Similar results were obtained by Sperling et al, who found neuropsychological data derived from intracarotid amobarbital testing significant for seizure control. ${ }^{36}$ Also in this study, neuropsychological profiles were helpful for decision making in approximately $60-70 \%$ of patients. A concordant and lateralising neuropsychological pattern contributed to the multifactorial prediction model for satisfactory seizure relief (Engel Classes I, II), but not for the prediction of complete seizure relief (Engel Class I). However, it is important to note that results from such statistical models are dependent on selection of factors and population, so that results cannot be interpreted as general evidence.

Magnetic resonance imaging plays a major role in the presurgical evaluation of epilepsy patients. ${ }^{6}{ }^{19}$ During the last two decades, increasingly more lesions have been detected with MRI in patients with TLE. ${ }^{4}$ Different MRI findings-for example, presence of mesial temporal sclerosis-have shown to be predictive for satisfactory seizure relief. ${ }^{35}{ }^{37-39}$ A majority of the MRI findings were classified as "lesions", because preoperative differentiation between developmental tumours and dysplastic lesions as well as between developmental tumours and glial tumours was uncertain in most cases. With developing experience of MRI interpretation, the rate of correct MRI diagnoses is clearly increased. ${ }^{6}$

\section{Histopathology}

Seizure control rates did not differ significantly with different histopathological diagnoses. However, excellent seizure relief rates were obtained with gangliogliomas and DNTs. It is known from previous publications that epilepsy surgery for these developmental tumours has an excellent prognosis with respect to seizure control. ${ }^{603031}$ Success rates for "ordinary gliomas" are less promising, probably because of the infiltrative nature of these tumours. ${ }^{60}$ It is widely accepted that seizure relief is less frequent after surgery for malformative lesions (for example, cortical dysplasias). No patient with MRI diagnosed periventricular nodular heterotopias was postoperatively seizure free as described by $\mathrm{Li}$ 
et al ${ }^{40}$ Cortical dysplasias are known to be associated with widespread changes of cortical architecture, which can be only partly detected with MRI studies. ${ }^{41}{ }^{42}$ Our approach with limited resections, focusing on a morphologically identified lesion, does not permit an estimation of the rate of more widespread histopathological alterations in the temporal lobe. It is interesting to note that dysplastic malformations were operated with an $80 \%$ chance of good seizure control. One may speculate whether mesiotemporal dysplasias involve only circumscribed regions and might be restricted to limbic areas. Complete resection based on MRI criteria was shown to result in very satisfying seizure relief rates. ${ }^{43}$ Future studies may clarify differences between dysplastic malformations in different cerebral areas. ${ }^{43} 44$

\section{Resection strategies}

The question of how to resect an epileptogenic focus or lesion in order to achieve seizure control is still matter of discussion. Anterior temporal lobectomy is the most widely performed standard resection for TLE, and most of the outcome results have been obtained with this procedure. ${ }^{4}{ }^{15}$ Nevertheless, beneficial effects-especially regarding postoperative neuropsychological results - have been described for more limited

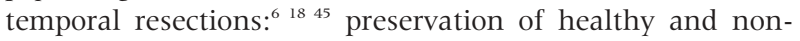
epileptogenic brain tissue may improve postoperative function. Similar rates of seizure relief and side effects were obtained with ATL and AH for the treatment of MTLE with AHS. ${ }^{6}{ }^{46}{ }^{47}$ There are different approaches in order to limit the extent of resection: "intraoperative tailoring" with electrocorticography-for example, to determine the length of hippocampal resection, ${ }^{48}$ or "preoperative tailoring" with guidance by imaging data and results from preoperative monitoring, eventually with chronically implanted electrodes. ${ }^{19}{ }^{22}$ A potential benefit of intraoperative MRI is still under investigation. ${ }^{49}$

Patients presented in this study were operated under the regime of "preoperative tailoring": complete resections of lesions demonstrated with MRI was primarily intended, if possible. The resection was extended-for example, with a "complete" hippocampectomy-whenever clinical or electrophysiological data suggested a seizure onset in the respective distant areas. The practice of this approach is mainly dependent on elaborate preoperative imaging, which has become possible with advances in MRI technology during the last 15 years..$^{50}$ "Preoperative tailoring" resulted in five different classes of approaches to treat lesional MTLE (fig 1), which resulted in similar rates of seizure relief. However, satisfactory seizure control was only obtained in one of three patients with TEMPAH. One may speculate whether anterior temporal lobectomy might be more successful in patients with mesial lesions, which extend into the lateral parts of the temporal lobe.

We feel encouraged that "preoperative tailoring" is a suitable approach in lesional MTLE. However, standard ATL might have resulted in even better seizure control rates. Whether patients have a benefit regarding their neuropsychological performance cannot be answered with this study. It has to be noted that lesional MTLE accounts only for a relatively small subgroup of TLE patients, so that controlled studies with respect to seizure control and neuropsychological performances are difficult to realise. Surgical procedures applied here proved to be safe, neurological complications were mainly temporary: only two patients suffered mild permanent deficits.

In conclusion, elaborate MRI, clinical, neuropsychological, and electrophysiological investigations are necessary features in the presurgical evaluation of lesional MTLE. As well as the obligatory presence of a mesiotemporal lesion, here, a model with four factors supportive for satisfactory seizure control comprised: seizure onset after 10 years of age, presence of complex partial seizures, absence of a neurological deficit or mental retardation, and a correlating lateralising neuropsychological deficit. The strategy of "preoperative tailoring" for lesional MTLE has proved to be safe and provides a good chance for satisfactory seizure relief.

\section{ACKNOWLEDGEMENT}

We wish to thank the neurosurgeons D Van Roost, B Meyer, J Zentner, and E Behrens. We wish to thank C Luyken, P Süßmann, and P Stausberg for supporting data collection and documentation.

\section{Authors' affiliations}

H Clusmann, T Kral, E Fackeldey, J Schramm, Department of Neurosurgery, University Bonn Medical Centre, Bonn, Germany I Blümcke, Department of Neuropathology, University Bonn Medical Centre, Bonn, Germany

C Helmstaedter, J von Oertzen, Department of Epileptology, University Bonn Medical Centre, Sigmund-Freud-Str. 25, 53105 Bonn, Germany H Urbach, Department of Neuroradiology University Bonn Medical Centre, Bonn, Germany

Supported by grants from the Deutsche Forschungsgemeinschaft (German Research Council), Sonderforschungsbereich 400 Subprojekt B1 to CE Elger/J Schramm and Transregio TR3, Subproject C5 (D Dietrich, T Kral, W Mueller) to by two grants of the University Bonn Medical Centre (Bonfor) to J Schramm and H Clusmann/T Kral.

\section{REFERENCES}

1 Hamer HM, Najm I, Mohamed A, et al. Interictal epileptiform discharges in temporal lobe epilepsy due to hippocampal sclerosis versus medial temporal lobe tumors. Epilepsia 1999;40:1261-8.

2 Salanova V, Markand O, Worth R. Temporal lobe epilepsy surgery: outcome, complications, and late mortality rate in 215 patients. Epilepsia 2002;43:170-4.

3 Schulz R, Luders HO, Hoppe M, et al. Interictal EEG and ictal scalp EEG propagation are highly predictive of surgical outcome in mesial temporal lobe epilepsy. Epilepsia 2000;41:564-70.

4 Radhakrishnan K, So EL, Silbert PL, et al. Predictors of outcome of anterior temporal lobectomy for intractable epilepsy: a multivariate study. Neurology 1998;51:465-71.

5 Jeong SW, Lee SK, Kim KK, et al. Prognostic factors in anterior temporal lobe resections for mesial temporal lobe epilepsy: multivariate analysis. Epilepsia 1999;40:1735-9.

6 Clusmann H, Schramm J, Kral T, et al. Prognostic factors and outcome after different types of resection for temporal lobe epilepsy. J Neurosurg 2002;97:1131-41

7 Yasargil MG. Intracranial microsurgery. Clin Neurosurg 1970;17:250-6.

8 Behrens E, Schramm J, Zentner J, et al. Surgical and neurological complications in a series of 708 epilepsy surgery procedures. Neurosurgery 1997;41:1-9.

9 Wiebe S, Blume WT, Girvin JP, et al. A randomized, controlled trial of surgery for temporal-lobe epilepsy. N Engl J Med 2001;345:311-18.

10 Bien CG, Kurthen M, Baron K, et al. Long-term seizure outcome and antiepileptic drug treatment in surgically treated temporal lobe epilepsy patients: a controlled study. Epilepsia 2001;42:1416-21.

11 Hennessy MJ, Elwes RD, Rabe-Hesketh S, et al. Prognostic factors in the surgical treatment of medically intractable epilepsy associated with mesia temporal sclerosis. Acta Neurol Scand 2001;103:344-50.

12 Mohamed A, Wyllie E, Ruggieri P, et al. Temporal lobe epilepsy due to hippocampal sclerosis in pediatric candidates for epilepsy surgery. Neurology 2001;56:1643-9.

13 Zentner J, Hufnagel A, Wolf HK, et al. Surgical treatment of temporal lobe epilepsy: clinical, radiological, and histopathological findings in 178 patients. J Neurol Neurosurg Psychiatry 1995;58:666-73.

14 lannelli A, Guzzetta F, Battaglia D, et al. Surgical treatment of temporal tumors associated with epilepsy in children. Pediatr Neurosurg 2000;32:248-54

15 Hennessy MJ, Elwes RD, Honavar M, et al. Predictors of outcome and pathological considerations in the surgical treatment of intractable epilepsy associated with temporal lobe lesions. J Neurol Neurosurg Psychiatry $2001 ; 70: 450-8$.

16 Ho SS, Berkovic SF, McKay WJ, et al. Temporal lobe epilepsy subtypes: differential patterns of cerebral perfusion on ictal SPECT. Epilepsia 1996:37:788-95.

17 Mclntosh AM, Wilson SJ, Berkovic SF. Seizure outcome after temporal lobectomy: current research practice and findings. Epilepsia 2001;42:1288-307.

18 Helmstaedter C, Elger CE, Hufnagel A, et al. Different effects of left anterior temporal lobectomy, selective amygdalohippocampectomy, and temporal cortical lesionectomy on verbal learning, memory, and recognition. J Epilepsy 1996;9:39-45. 
19 Kral T, Clusmann $\mathrm{H}$, Urbach $\mathrm{H}$, et al. Preoperative evaluation for epilepsy surgery (Bonn algorithm). Zentralb/ Neurochir 2002;63:106-10.

20 Zentner J, Hufnagel A, Wolf HK, et al. Surgical treatment of neoplasms associated with medically intractable epilepsy. Neurosurgery 1997:41:378-86.

21 Zentner J, Wolf HK, Ostertun B, et al. Gangliogliomas: clinical, radiological, and histopathological findings in 51 patients. J Neurol Neurosurg Psychiatry 1994;57:1497-502.

22 Behrens E, Zentner J, Van Roost D, et al. Subdural and depth electrodes in the presurgical evaluation of epilepsy. Acta Neurochir 1994;128:84-7.

23 Van Roost D, Solymosi L, Schramm J, et al. Depth electrode implantation in the length axis of the hippocampus for the presurgical evaluation of medial temporal lobe epilepsy: a computed tomography-based stereotactic insertion technique and its accuracy. Neurosurgery 1998;43:819-26.

24 Helmstaedter C, Kurthen M, Lux S, et al. Temporal lobe epilepsy: longitudinal clinical, neuropsychological and psychosocial follow-up of surgically and conservatively managed patients [in German]. Nervenarzt 2000;71:629-42.

25 von Oertzen J, Urbach H, Jungbluth S, et al. Standard magnetic resonance imaging is inadequate for patients with refractory focal epilepsy. J Neurol Neurosurg Psychiatry 2002;73:643-7.

26 Yasargil MG, Teddy PJ, Roth P. Selective amygdalo-hippocampectomy. Operative anatomy and surgical technique. Adv Tech Stand Neurosurg 1985; 12:93-123.

27 Wolf HK, Campos MG, Zentner J, et al. Surgical pathology of temporal lobe epilepsy. Experience with 216 cases. J Neuropathol Exp Neurol 1993:52:499-506.

28 Blümcke I, Giencke K, Wardelmann E, et al. The CD34 epitope is expressed in neoplastic and malformative lesions associated with chronic, focal epilepsies. Acta Neuropathol (Berl) 1999;97:481-90.

29 Engel JJ, Van Ness P, Rasmussen TB, et al. Outcome with respect to epileptic seizures. In: Engel JJ, ed. Surgical treatment of the epilepsies. New York: Raven Press Ltd, 1993:609-21.

30 Morris HH, Matkovic Z, Estes ML, et al. Ganglioglioma and intractable epilepsy: clinical and neurophysiologic features and predictors of outcome after surgery. Epilepsia 1998;39:307-13.

31 Raymond AA, Halpin SF, Alsanjari N, et al. Dysembryoplastic neuroepithelial tumor. Features in 16 patients. Brain 1994;117:461-75.

32 Abou-Khalil B, Andermann E, Andermann F, et al. Temporal lobe epilepsy after prolonged febrile convulsions: excellent outcome after surgical treatment. Epilepsia 1993;34:878-83

33 Schramm J, Kral T, Grunwald T, et al. Surgical treatment for neocortical temporal lobe epilepsy: clinical and surgical aspects and seizure outcome. J Neurosurg 2001;94:33-42.

34 Foldvary N, Nashold B, Mascha E, et al. Seizure outcome after temporal lobectomy for temporal lobe epilepsy: a Kaplan-Meier survival analysis. Neurology 2000;54:630-4.
35 Holmes MD, Born DE, Kutsy RL, et al. Outcome after surgery in patients with refractory temporal lobe epilepsy and normal MRI. Seizure 2000;9:407-11. 36 Sperling MR, Saykin AJ, Glosser G, et al. Predictors of outcome after anterior temporal lobectomy: the intracarotid amobarbital test. Neurology 1994:44:2325-30.

37 Antel SB, Li LM, Cendes F, et al. Predicting surgical outcome in temporal lobe epilepsy patients using MRI and MRSI. Neurology 2002;58:1505-12.

38 Gilliam F, Bowling S, Bilir E, et al. Association of combined MRI, interictal EEG, and ictal EEG results with outcome and pathology after temporal lobectomy. Epilepsia 1997;38:1315-20.

39 Kuzniecky R, Burgard S, Faught E, et al. Predictive value of magnetic resonance imaging in temporal lobe epilepsy surgery. Arch Neurol 1993;50:65-9.

40 Li LM, Dubeau F, Andermann F, et al. Periventricular nodular heterotopia and intractable temporal lobe epilepsy: poor outcome after temporal lobe resection. Ann Neurol 1997;41:662-8.

41 Sisodiya SM, Free SL, Stevens JM, et al. Widespread cerebral structural changes in patients with cortical dysgenesis and epilepsy. Brain 1995:118:1039-50

42 Hardiman O, Burke T, Phillips J, et al. Microdysgenesis in resected temporal neocortex: incidence and clinical significance in focal epilepsy. Neurology 1988;38: 1041-7.

43 Urbach H, Scheffler B, Heinrichsmeier T, et al. Focal cortical dysplasia of Taylor's balloon cell type: a clinicopathological entity with characteristic neuroimaging and histopathological features, and favorable postsurgical outcome. Epilepsia 2002;43:33-40.

44 Barkovich AJ, Kuzniecky RI, Jackson GD, et al. Classification system for malformations of cortical development: update 2001. Neurology 2001:57:2168-78.

45 Katz A, Awad IA, Kong AK, et al. Extent of resection in temporal lobectomy for epilepsy. II. Memory changes and neurologic complications. Epilepsia 1989;30:763-71.

46 Arruda F, Cendes F, Andermann F, et al. Mesial atrophy and outcome after amygdalohippocampectomy or temporal lobe removal. Ann Neurol 1996;40:446-50

47 Egan RA, Shults WT, So N, et al. Visual field deficits in conventional anterior temporal lobectomy versus amygdalohippocampectomy. Neurology 2000;55:1818-22.

48 McKhann GM, Schoenfeld-McNeill J, Born DE, et al. Intraoperative hippocampal electrocorticography to predict the extent of hippocampal resection in temporal lobe epilepsy surgery. J Neurosurg 2000;93:44-52.

49 Buchfelder M, Fahlbusch R, Ganslandt O, et al. Use of intraoperative magnetic resonance imaging in tailored temporal lobe surgeries for epilepsy. Epilepsia 2002;43:864-73.

50 Berkovic SF, Mclntosh AM, Kalnins RM, et al. Preoperative MRI predicts outcome of temporal lobectomy: an actuarial analysis. Neurology 1995;45:1358-63. 